\title{
Electric Vehicle Development and Low-Carbon Transport in Vietnam
}

\author{
Le Anh Tuan ${ }^{(\bowtie)}$ \\ Hanoi University of Science and Technology, Hanoi, Vietnam \\ tuan.1eanh@hust.edu.vn
}

\begin{abstract}
Vietnam's social and economic development achievements are remarkable. However a steep rise in income and economic growth has led to rapid motorization and high energy demand. The transport sector is a major consumer of energy in Vietnam and thus it is one of the key sectors which produces most emissions including green house gas (GHG). As a result, according the emissions per GDP, Vietnam is ranked the $13^{\text {th }}$ most carbon intensive economy in the world, and $4^{\text {th }}$ among the low- and middle- income countries in East Asia. GHG emissions from the transport sector are expected to triple by 2030, to nearly 90 million tons carbon dioxide equivalent (CO2e).

In road transport sector, there are about 40 million vehicles, including about 35 million motorbikes, over around 96 million population. Almost all of road vehicles use internal combustion engines which emit high GHG and high toxic emissions. A pathway for low-carbon transport is crucial, consisting the shift from conventional vehicles to electric ones.

This talk addresses the global electric vehicle outlook, related technologies of electric vehicles, Vietnam current situation of automotive industry and contribution of low-carbon transport scenarios, in general, and of electric vehicles, in particular, to GHG reduction for Vietnam.
\end{abstract}

Keywords: Vehicle outlook $\cdot$ Electric vehicle development $\cdot$ Low-carbon transport 